\title{
Static vs Dynamic Architecture of Aware Cyber Physical Systems of Systems
}

\author{
Peter Bernus \\ Institute for Integrated and Intelligent Systems (IIIS) \\ Griffith University \\ Brisbane, QLD, Australia \\ orcid.org/0000-0001-5371-8743
}

\author{
Ovidiu Noran \\ Institute for Integrated and Intelligent Systems (IIIS) \\ Griffith University \\ Brisbane, QLD, Australia \\ orcid.org/ 0000-0002-2135-8533
}

\begin{abstract}
The Enterprise Architecture and Systems Engineering communities are often faced with complexity barriers that develop due to the fact that modern systems must be agile and resilient. This requires dynamic changes to the system so as to adapt to changing missions as well as changes in the internal and external environments. The requirement is not entirely new, but practitioners need guidance on how to manage the life cycle of such systems. This is a problem because we must be able to architect systems by alleviating the difficulties in systems life cycle management (e.g., by helping the enterpriseor systems engineer organise and maintain models and architecture descriptions of the system of interest). Building on Pask's conversation theoretic model of aware (human or machine) individuals, the paper proposes a reference model for systems that maintain their own models real time, act efficiently, and create system-level awareness on all levers of aggregation.
\end{abstract}

Keywords-Systems Engineering, Enterprise Engineering, Model-based Systems Engineering, Awareness

\section{INTRODUCTION}

The Enterprise Architecture and Systems Engineering communities are often faced with complexity barriers that develop because modern systems must be agile and resilient, which requires dynamic changes to the system so as to adapt to changing missions as well as changes and influences originating from the internal and external environments.

This requirement is not entirely new; but practitioners still need guidance on how to manage the life cycle of such systems using the architecture framework (AFs) used in their domain. The lack of specific guidance regarding the development of agile and resilient systems is problematic as AFs have the explicit purpose to assist the management of a system's life cycle by helping the enterprise- or systems engineer organise models and architecture descriptions of the system of interest.

Traditionally, these models are produced in the initial stage/s of a system's life - cf. Model Based Systems Engineering (MBSE) - but models are increasingly being used for model-based control, and for supporting various management \& control and service functions during system operation, partial or total decommissioning, or during maintenance, repair and overhaul.

The term 'digital twin' [8] [16] has been popularised as the set of models collectively mirroring the System of Interest in real time during operation [11]. This conceptual development took place over many years - e.g., since long time databases have been maintaining a real time mirror of relevant facts in the physical world, and by taking snapshots they also mirrored the history of these facts for management decision support, business intelligence, and other purposes.

Process management systems also became popular [24] and allowed the enterprise to maintain a real time mirror of some process instances (and history traces), and these could be used for improved management and control, as well as to inform and support process improvement practices.

Today, the concept of digital twin potentially covers the entire enterprise in scope, including physical equipment, humans and software \& hardware alike. Importantly, this coverage also includes the products and product lines as entities of interest, as well as the relevant elements of the environment, and the relationships among all of the above. After the concept was thus generalised, it found applications in product life cycle management [11], logistics [21], modelbased control [19], predictive maintenance [12], and supply chain management [7]) - to name a few. Technologies that support this development include the Internet of Things (sensor networks, actuators, etc.), and various computational techniques, such as machine learning, data analytics, and associated technologies capable of handling large amounts of heterogeneous data (e.g., data lakes [14]).

The emphasis of this paper is whether current architecture practice is sufficiently supportive of these developments. Unfortunately, a) the perception is that existing architecture frameworks are not well prepared to support this trend, and b) even if they do, practitioners need a demonstration of how current AFs can help them architect and develop such systems. The authors believe that the above issues are important because a 'digital twin methodology' should start from a representation of the engineering and management problems that result from the requirement that the system of interest must be agile and resilient. The paper is therefore focused on issue b) by demonstrating how such systems can be modelled using existing AFs, thereby giving methodological handles for architects.

The authors shall make use of the GERAM framework, which is part of the ISO standard ISO15704:2019 on 'Enterprise Modelling and Architecture' [6]. This AF was selected in preference to other AFs because i) it has a built-in modelling framework (GERA), and ii) has an explicit representation of relationships among the life-cycles of multiple entities of interest, whereupon all other AFs are based on architecture descriptions that can be derived as views from underlying models, iii) according to the NIST 'Standards Landscape for Smart Manufacturing'[13], ISO15704 is the top-level enterprise architecture standard for Smart Manufacturing (see Fig. 1). 


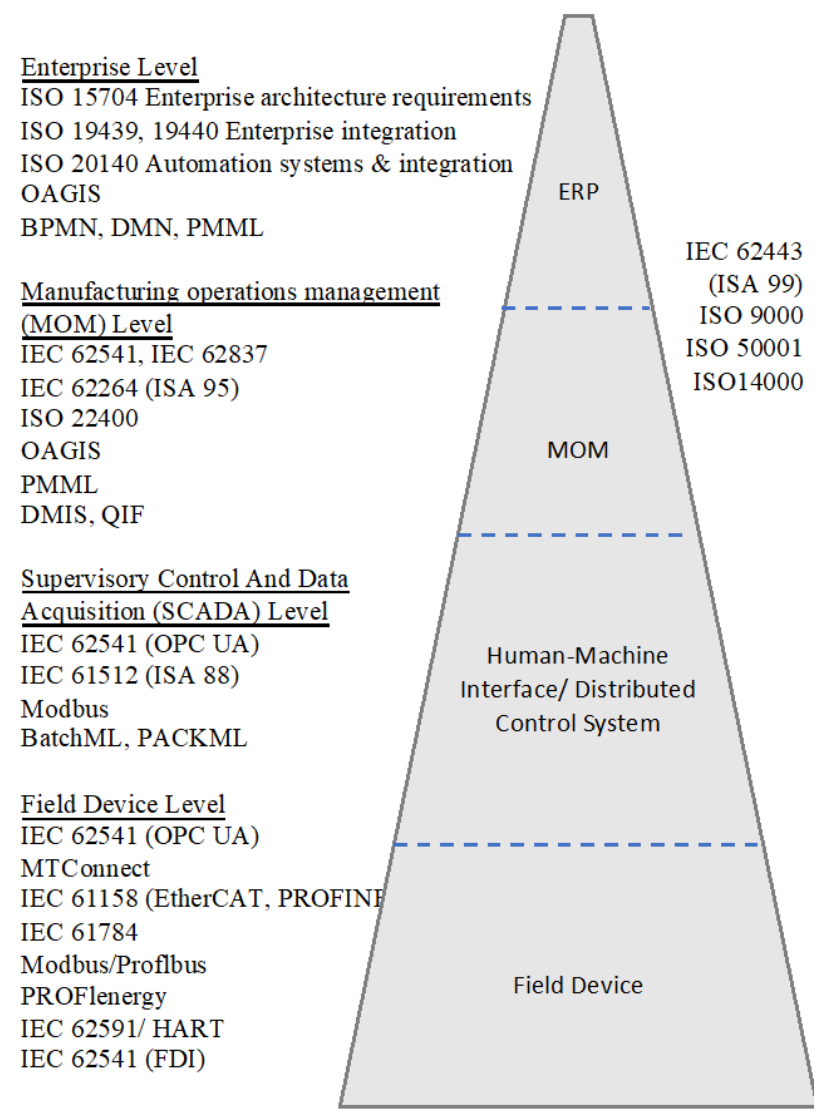

Fig. 1. NIST Standards Landscape for Smart Manufacturing (redrawn after [13])

The authors shall also make use of the main systems engineering process standard, ISO15288 [5], in order to maintain the conceptual alignment between Enterprise Architecture and the Architecture of Systems (as systems engineering defines it).

This paper is therefore about the underlying models, their dynamics and evolution, leaving the derivation of architecture descriptions (for the consumption of various stakeholders) to the practitioner and the tools supporting them.

A word of caution before reading on: this paper adheres to the terminology of ISO15704/GERAM, and if the reader is not thoroughly familiar with those concepts, that can be the source of several misunderstandings regarding concepts such as life cycle, life history, life cycle phase, life history stage, life cycle relationships.

Therefore, for the sake of conciseness and for the benefit of the reader who already knows ISO15704/GERAM, these concepts are summarised and explained in the Appendix.

\section{ARCHITECTURE MODELS AND THEIR RELATIONSHIPS}

ISO15704 differentiates between various types of entities in the enterprise, each having its life cycle (entities like products, projects, programmes, organisations, and various virtual enterprises).

The concept of life cycle in the standard is abstracting from time and consists of life cycle activity types, such as identification, concept development, requirements, preliminary- and detailed design, implementation /build, (configure \& deploy), operation and decommissioning.

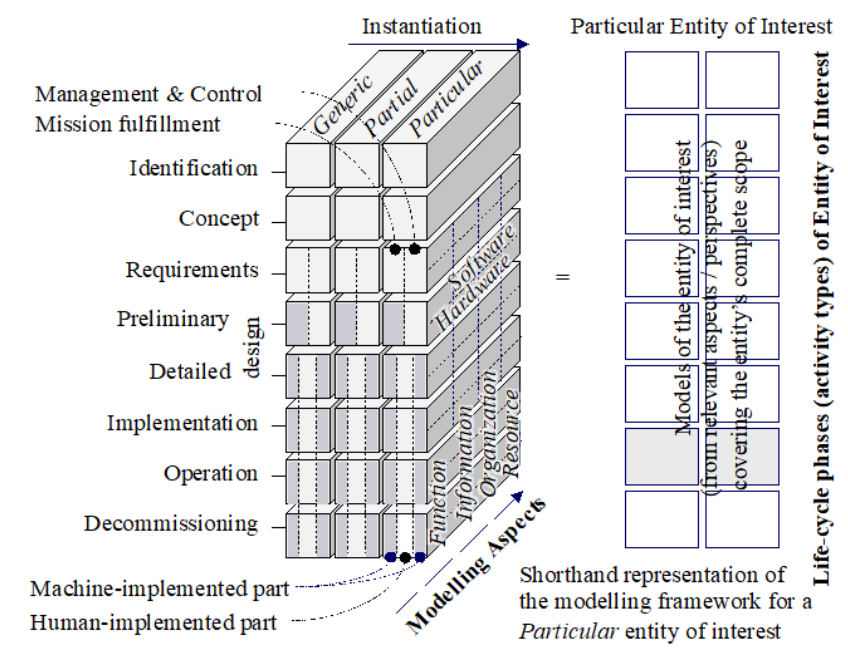

Fig. 2. ISO15704/GERAM's modelling framework. The framework may be populated by models for a particular entity, but also with reference models ('partial models') and with generic models (ontologies / metamodels) of the domain

i) Entity A Entity B LC activity types ii) Entity B
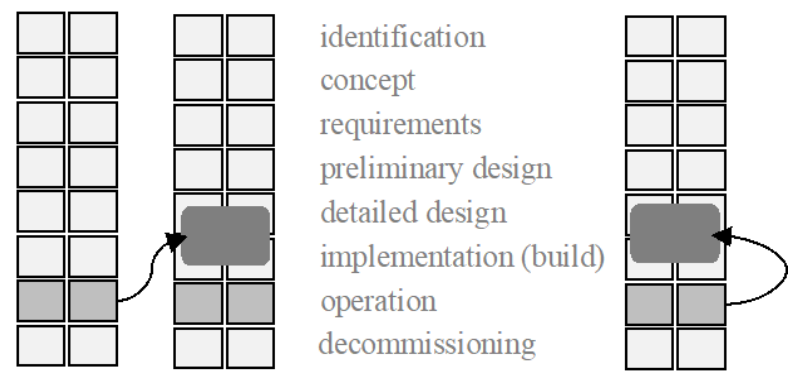

Fig. 3. Example of life cycle relationship between two entities: either i) 'A' performs the detailed design and building of ' $\mathrm{B}$ ', or ii) ' $\mathrm{B}$ ' performs its own detailed design and building activities and maintains the models of itself (displaying a form of adaptation and agility)

The temporal aspect is subsequently covered as a collection of life cycle activity instances that can be grouped into 'sequences of events' separated by milestones.

The time periods between such milestones are called life history stages (corresponding to what ISO15288 calls 'life cycle stages'). Consequently, the outputs of life cycle activity types are model types, which are then instantiated during the life history and evolution of the system of interest (with a version history).

Using GERAM's modelling framework (see Fig. 2) the systems engineer can arrange the models for each enterprise entity separately. With the progression of the enterprise's life, some entities are created and some are decommissioned, and each has a life history of its own.

E.g., the 'Supporting Entities' in ISO15288 refer to systems that contribute to the life of the enterprise in various stages of its life. The relationship between the life cycles of entity A and B can be represented (as a shorthand) by drawing an arrow from the operation of $\mathrm{A}$ to those life cycle activities of B, to which A contributes (see Fig. 3). Accordingly, a long chain of life cycle relationships may exist - see Fig. 4. 


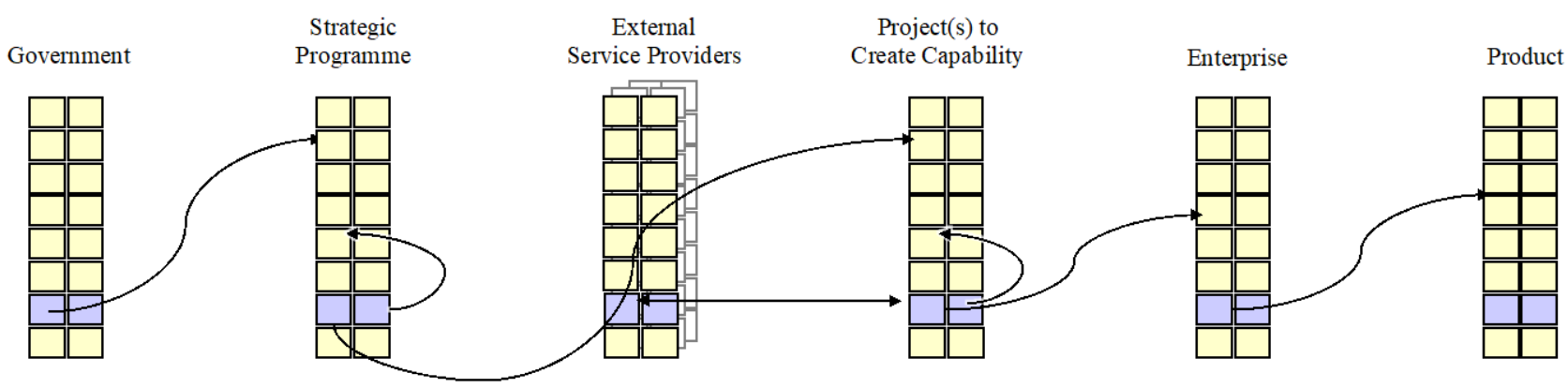

Fig. 4. Illustration of a long chain of life cycle relationships (note the self-generating nature of programme and project entities)

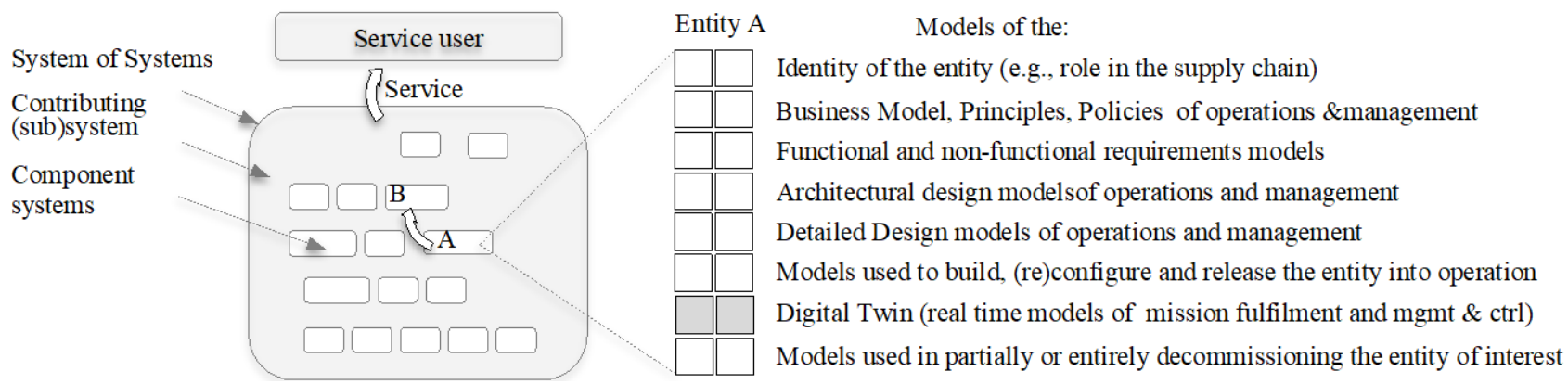

Fig. 5. Models populating the Modelling Framework during the creation vs. during the operation of an entity. (In a layered System of Systems each component may be operated by a different entity and has its own life cycle.)

A more elaborate example is where life cycle relationships exist between the Enterprise and the Virtual Entities dynamically created by that Enterprise (such as Programmes, Projects, Service Virtual Enterprises, etc.), as well as Products (which latter can be a complex entity in its own right, such as a ship or a factory).

Note that Projects and Programmes are 'virtual entities (VEs)' in the sense that most of the mission fulfilment and management processes that make up the VE's Operation are performed by other entities, and their contribution(s) are coordinated by these processes.

Using this 'VE pattern', complex coordinated operations (such as in Programmes, Projects, military Missions, etc.) can be architected as separate systems in their own right, reducing the design complexity of the generating entities (i.e., of the participating enterprises). This may be achieved if the management processes of the VEs include self-generating / self-reconfiguring capabilities (see Fig. 3/ii). This is what will be used further in Section III.

For example, a government department may be responsible for creating a Programme, which is then responsible for creating Projects, that in turn create a capability in the enterprise that creates the Product, etc.

Furthermore, Enterprise Networks can also be represented as VEs, with members contributing to its mission fulfilment and management processes, and the Network in turn may create other VEs in a chain (such as collaborative projects, or virtual service entities).

Fig. 5 arranges (along the atemporal life cycle dimension) the models created during the design, building and (re)configuration of an entity, as well as its models maintained during Operation. These models collectively constitute the 'digital twins' of that entity.
In the life of any entity, there are models capturing the so-called 'static architecture' that is immutable in the sense that modifying these constitute a major change to the entity - e.g., by significantly altering its capability(ies) essentially changing the entity's identity. Conversely, the so-called 'Dynamic architecture' results from adaptively configuring the entity, and releasing it into operation. (This routinely happens in manufacturing when a workshop is (re)configured to produce a new product mix, or adapts to sudden changes in resource availability.)

In case of self-configuring, the entity must have such processes as part of its long-lived ('static') architecture, so as to be able to generate the requisite dynamic structures. Note that all of the above recursively applies to all levels of composition (layers and aggregates) in a System of Systems (SoS).

\section{PATTERNS FOR ARCHITECTING AWARE SYSTEMS}

Intuitively, an entity maintaining its own models may be construed as a system that is aware of itself. However, in order to claim to have created an architecture of aware systems, a theoretical underpinning is necessary. Therefore, the authors apply a theory that explains what are the fundamental elementary building blocks of aware systems, whether natural, artificial or hybrid.

This was originally proposed by Pask [20] for learning systems, who demonstrated a minimal set of processes (called a 'conversational skeleton', or pattern) that are necessary to display awareness (see Fig. 6). The two sides of the figure illustrate the participating processes in a socalled 'conversation', and both sides have minimum two levels. Level $_{0}$ is the set of processes that participants ' $a$ ' and ' $b$ ' use to perform some actions in the domain of interest, and are connected through an 'interface' that both sides can observe and manipulate. Any form of such externalisation 
is admissible (observable demonstration, or communication using one or more languages $\left[\mathrm{L}_{0}\right.$ and $\left.\mathrm{L}_{1}\right]$ ).

Level $_{0}$ processes can collaborate performing tasks that achieve a common goal, or one side (using $\mathrm{L}_{0}$ ) can demonstrate to the other the performance of a task, while the other side's Level ${ }_{0}$ observes.

Level $l_{1}$ includes two sets of contextual processes $\mathrm{Pa}^{1}$ and $\mathrm{Pb}^{1}$, with three roles:

- using the interface, they establish context in a conversation, which includes the common understanding of a goal, or concept, and a commitment to follow a course of action;

- communicate while acting cooperatively to progress the two sides' Level 0 processes;

- furthermore, Level ${ }_{1}$ may be used by one side to instruct or teach the other side, or use 'teach back' to prove that common understanding has been achieved. Therefore, Level $_{1}$ includes the set of processes able to create, invoke, modify and observe the progression of Level $_{0}$ processes of their respective sides.

Pask called this set of four processes a Psychological Individual (henceforth P-Individual).

There exist multiple possibilities of embodying a Pindividual in physical individuals (human, machine, or hybrid agents). Some possibilities are illustrated in Fig. 7.

Furthermore, these individuals can be dynamically combined into Emerging Individuals - i) by composition and abstraction (creating an emergent agent out of multiple cooperating agents) (see Fig. 7c), or ii) by dynamically creating and re-creating a control hierarchy (holarchy) that suits the situation [15][23].

There is an opportunity, which literature does not seem to have explored yet, namely, that there exists a relationship between models of P-individuals and physical individuals (agents), and models of situation awareness (SAW).

To this end, we use Endsley's early work that established a functional model of processes involved in creating situation awareness (SAW) [1], and the contribution of Niklasson et al. [17] to the theory of shared SAW, as these can be further elaborated using conversation theory.

E.g., it can be demonstrated what processes are necessary for a P-individual to create individual SAW on the one hand, and in conversation with others a shared SAW on the other, as well as what additional conditions are necessary for physical individuals to achieve the same.

Fig. 8 illustrates the sharing of SAW using a Conversation. The terminology 'being aware with' (see Fig. $7 b)$ may sound unusual; it is an expression (coined by Pask) aiming to stress the fact that i) if we take the two sides as being implemented by two agents, then neither of the parties will have SAW without the other, however, ii) if we take the $\mathrm{P}$-individual as being implemented in one agent, then that agent must implement the two sides of the conversation.

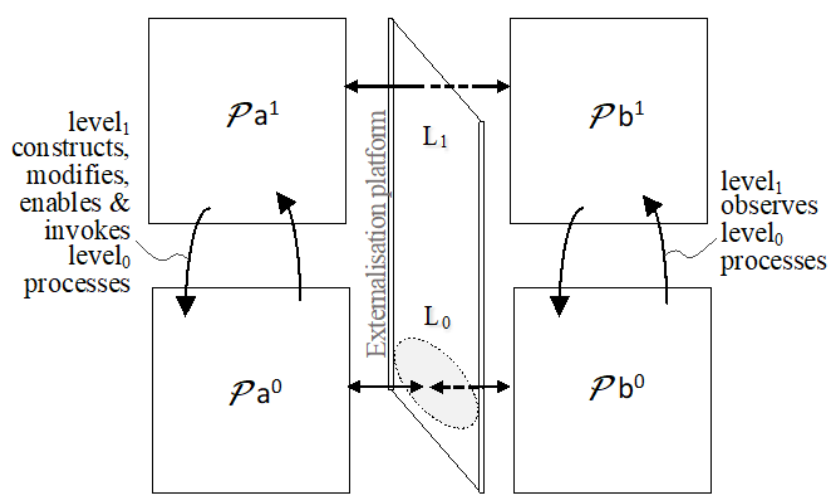

Fig. 6. Conversation theoretic process pattern (called a 'P-Individual')

(a) Aware agent

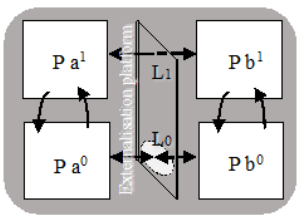

(b) Two agents $\mathrm{A}$ and $\mathrm{B}$ (A only aware with $\mathrm{B}$ ):

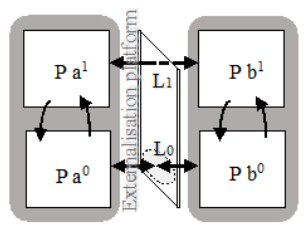

(c) Two agents A and B with common understanding, but both have individual awareness as well:

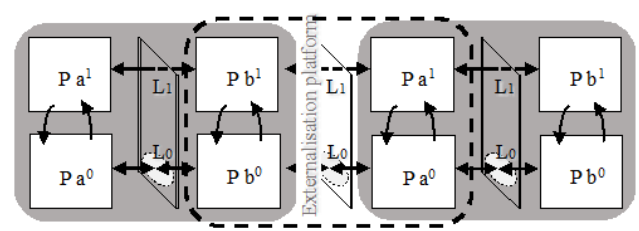

Fig. 7. Multiple ways of physically manifesting P-individuals. (Grey boxes are physical individuals; dotted box represents an emergent agent that in itself is a P-individual.)

This results in the ability of an agent to make sense of the situation on its own (see Fig. 7a) [1][4][22].

Some or all of these processes can be tacit (performed without awareness and very efficiently), even though for the external observer the entire set of processes appears to be performed in an aware way (i.e., awareness emerges).

In fact, as illustrated in Fig. 9, every one of the eight 'white boxes of processes' $\mathrm{Px}_{\mathrm{i}}$ (where ' $\mathrm{i}$ ' is the Level, and ' $x$ ' is the index running across the two sides of processes for each agent) can be subdivided into three more levels according to the nature of implementation [9].

Ostensibly the bottom (tacit) level in Fig. 9 is the only efficient way to implement processes in humans, whereupon in machines some efficient algorithms (pattern recognition, machine [deep] learning) are candidates, but procedural algorithmic implementations are often not good candidates. Notice: if the efficient tacit process produces ambiguity or unexpected outcome, the aware level must 'take over' so as to explain, interpret, project, and decide. 


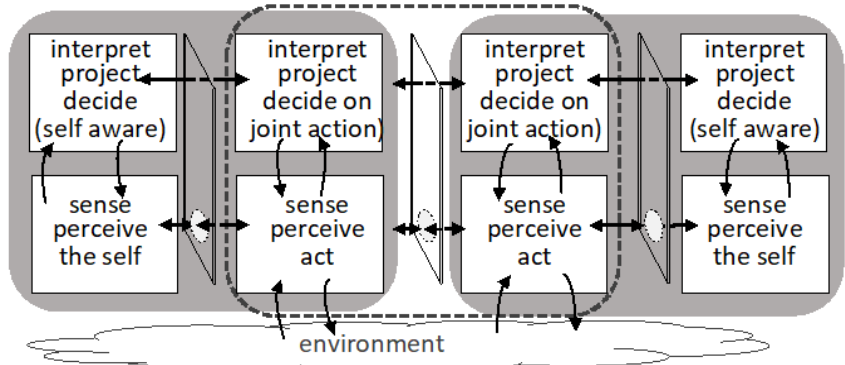

Fig. 8. Sharing SAW through conversation among two Physical individuals (agents) both implementing their own P-individual (and together implementing two sides of an emergent agent

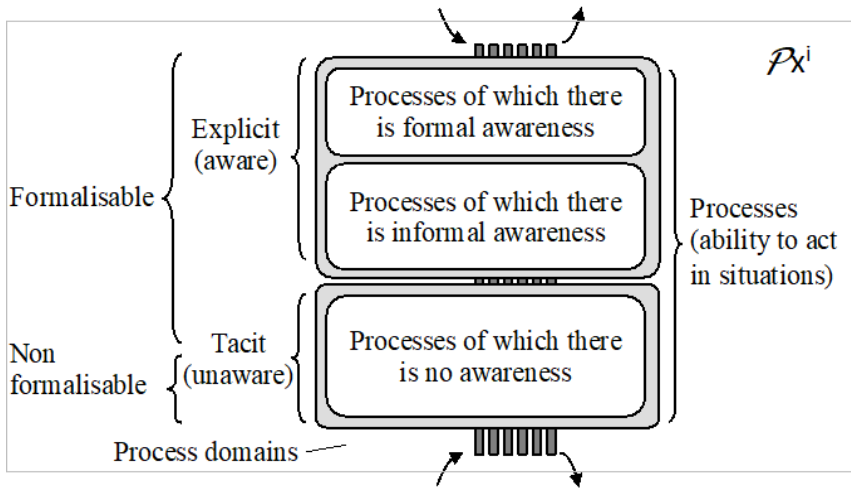

Fig. 9. Internal levels of knowledge (according to level of awareness) [9]. The bottom (tacit level) process type is the aim for efficiency reasons, but the model accounts for the need to learn new processes on Level ${ }_{0}$, and new situations on Level

The above model is recursive, i.e., it is a pattern that repeats on all aggregation levels. This aggregation does not have to be static: it can be dynamically (re)created, as an adaptation or optimisation measure in light of changing internal or external events. This is because through this conversation the two agents naturally create a higher-level agent that in turn may participate in further higher-level aggregates (see Fig. 10).

Given the possible volatility of the environment, several connections between the physical individual and the environment as well as with the 'externalisation platform' may be severed, e.g., the sense/perceive processes may be hindered by loss of visibility, noise, loss of connectivity, etc. However, if remaining within the limits of the expected necessary real time requirements of action, this is not necessarily a problem. For example, if the cooperative agents can not (or prefer not to) communicate, they can still observe the effects of the other's action in the environment. Based on the shared intent and action repertoire they may also perform two actions, one overt (effects of which others can observe) and one covert (that only takes effect with a delay that is too late to counteract when it becomes apparent). Examples exist both in the commercial and military environments [22].

In terms of the hierarchy of control, the P-Individual can shift levels, for example, the (until now) implicit 'intent' of cooperative action, and the implicit knowledge of a range of shared situation knowledge is due to a conversation that took place prior to action (see Fig. 10). The (two or more) agents on the left-hand side are the cooperating agents (c.f. Fig. 8) tasked with completing a mission, whereupon the agent on the right-hand side is the agent that establishes common intent with them (but does not necessarily participate in the cooperative action). Note that this requires that these cooperative agents engage in a conversation that is on $\mathrm{Level}_{2}$ (for them). This can be training, prior to action, but also debriefing, and accounts for learning new situations.

Another notable structure is when agent ' $A$ ' does not

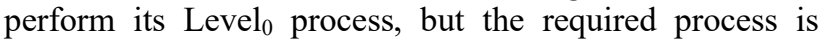
implemented by (outsourced to) another agent ' $B$ '. The implementation now requires that ' $A$ ' replace its Level $_{0}$ process with a process that is cooperating with agent ' $\mathrm{B}$ ', and a new P-individual is spawned, thereby ' $\mathrm{A}$ ' and ' $\mathrm{B}$ ' together implementing that P-individual. In other words, agents never directly control one another, but instead they negotiate in a conversation and agree to cooperatively perform a task (Fig. 10). The benefit is the additional resource and capability of ' $\mathrm{B}$ ' becoming available, but the cost is the need to communicate in an explicit fashion, which at times may negate the benefits of task sharing.

The present discussion assumes that cyber physical systems [10] are widely distributed, such as (for example) world-wide supply chains, that are in competition with other supply chains, and therefore must act in a manner akin to military operations and missions so as to remain adaptive, competitive, resilient and agile.

\section{THE SCOPE OF MODELS}

For the processes discussed in Section III to be able to generate useful information for decision making, they need sensory input, and must selectively perceive that input. This is the condition for developing (and maintaining) situation assessment (SA) and sensemaking (SAW) (sensing $\rightarrow$ perceiving $\rightarrow$ comprehending $\rightarrow$ projecting) [17][18].

The so-called 'dynamic business model' of a Cyber Physical System contains multiple entities of interest and their models (Fig. 4 and Fig. 5), each comprising many agents, but also every entity (ideally) acting as an emergent aware agent. The 'digital twin' of the physical system is comprised of these models, but with a caveat: the digital twin cannot be statically designed, because situation assessment and sensemaking will (possibly in real time) alter the focus of the sensory process and alter the selectivity of the perception.

In other words, the agent implementing the aware Pindividual must be directly invoking, enabling, constructing or modifying the set of sensory processes. Alternatively, if sensing is implemented by a separate individual agent, then create a P-individual to negotiate with that agent as illustrated in Fig. 7c.

The awareness of the cyber physical system boils down to not only having processes in the operation (that fulfil the mission of the system) and associated processes that implement the needed control functions, but also having processes that reason about these processes in context (and as a result change them in some way). Such change may involve improvement, reorganisation, reassignment of tasks, etc.

Example: Assume that a virtual enterprise (VE) was created by a network of companies to complete the design and delivery of a batch of products. The VE has its own management and control that tries to optimise the delivery of the goods given the changes that normally arise in a global supply chain. 


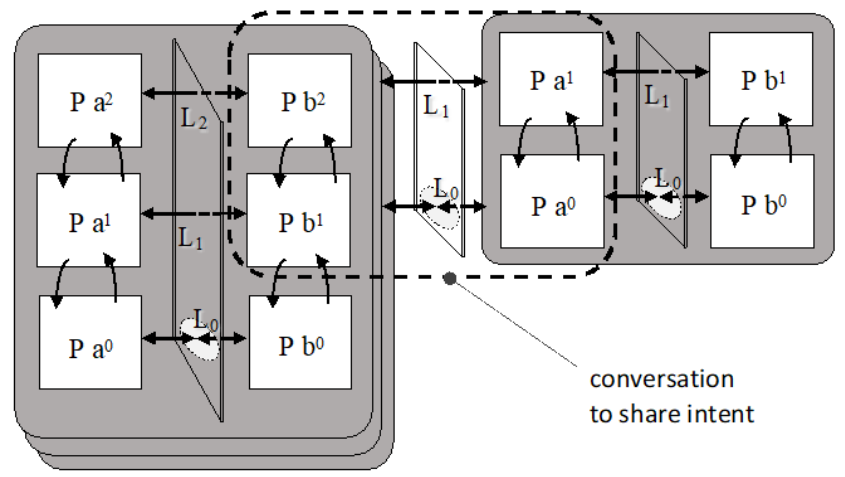

Fig. 10. Recursive process-pattern for aware entities

This management and control relies on two sets of models:

a) the models of the VE expressed as process- and associated information models, product models, as well as the models of the capabilities of the participants, including the VE's 'self',

b) the models (or other representations) of the process instances, resource status, product status / state, and the VE's self as a model instance, etc.

Together these models are forming the digital twins, perhaps shared through a data lake (allowing for all types of information, not only formal models).

As the situation in the supply chain changes, the VE's management \& control maintains awareness of the VE's status, and is able to reallocate resources, reroute deliveries, change production schedules, etc., effectively changing the $\mathrm{VE}$ in an adaptive way. The task is performed in a hierarchically distributed way, because each of the VE participants are in turn aware agents. This latter fact is important, as complete and centralised information sharing is impractical and often against commercial interests, i.e., information is still only shared on a need to know basis.

In addition to the above, a second level of management $\&$ control is exercised by the network of companies that created the VE in the first place. If the VE's management and control capabilities can not successfully resolve the consequences of the situation, or is unable to unambiguously resolve the information about the state of affairs, the network of companies as an agent would renegotiate or reconfigure the $\mathrm{VE}$ as the need dictates.

\section{CONCLUSION}

The paper followed a conceptual-analytical methodology, with the aim of developing a high-level model of cyber physical systems, that have awareness as a distinguishing feature. The model is inspired by Conversation Theory applied to cooperative multi-agent systems (including human, machine and hybrid agents).

Such systems are intrinsically more robust than systems managed using conventional control methods, and could be experimentally implemented using existing AI programming methods of two kinds (below), or by humans, or in a hybrid human-machine symbiosis:

a) Tacit processes implemented by humans or by machine learning / pattern recognition techniques, or purpose-built hardware - these are efficient, fast and do not rely on any sort of reasoning. While they are to some extent vulnerable, their efficiency makes them prime candidates for deployment.

b) Explicit processes that are performed in a rulebased / procedural manner. While they may be less efficient, they can be verified and used for training to eventually make the process tacit. Such robust, verifiable processes guard against the deployment or continued use of tacit processes if the situation is not appropriate for their use.

There is a danger of possible misinterpretation here: we stress that both a) and b) (tacit and explicit) may exist on each level of the conversation, including Level ${ }_{0}$ and Level ${ }_{1}$. However, due to the overwhelming need to act in the domain using tacit processes (for efficiency), it is expected that explicit processes on Level ${ }_{0}$ will predominantly be used during training, while Level $_{1}$ will require explicit processes for reasoning about situations (to create explainable situation awareness) [3].

Thus Level ${ }_{1}$ processes deploy, invoke, train and supervise Level ${ }_{0}$ processes, and this could be done in a tacit way, but when it comes to situation interpretation or projection, explicit processes will be needed.

The other novelty of the proposal is the combination of the architectural models of cyber-physical systems and of conversation theory with the considerations of knowledge domains, such as tacit, informal explicit and explicit.

In the future the authors aim at demonstrating the above model using multi-agent simulation techniques, with the dual goal of validating it as a reference architecture, and to demonstrate how agents could reason about forming emergent agents to maximise effectiveness and efficiency.

\section{REFERENCES}

[1] Bernus, P., Martin, R., Noran, O., Molina, A. (2021). The 25 years of the GERAM Framework. In IFIP $60^{\text {th }}$ Anniversary issue. Springer. (in press)

[2] Endsley, M. R. (2015). Situation awareness: operationally necessary and scientifically grounded. Cogn Tech Work, 17:163-167.

[3] Goranson, T., Cardier, B. (2013). A Two-sorted Logic for Structurally Modeling Systems. Progress in biophysics and molecular biology, 113:141-178

[4] Hong, M.-T., Benjamin, J. J., Müller-Birn, C. (2018). Coordinating Agents: Promoting Shared Situational Awareness in Collaborative Sensemaking. In Proc. ACM Conf. on Computer Supported Cooperative Work and Social Computing, Jersey City, NJ, USA.

[5] ISO/IEC. (2018). ISO/IEC 15288: Systems and software engineering - System life cycle processes.

[6] ISO/IEC. (2019). GERAM. In ISO15704-2019: Enterprise modelling and architecture - Requirements for enterprisereferencing architectures and methodologies.

[7] Ivanov D., Dolgui A., Das A., Sokolov B. (2019). Digital Supply Chain Twins: Managing the Ripple Effect, Resilience, and Disruption Risks by Data-Driven Optimization, Simulation, and Visibility. In: Handb. Ripple Effects in the Supply Chain. Int. Ser. Op. Research \& Management Science, 276. Springer, pp309-332.

[8] Jones, D., Snider, C., Nassehi, A., Yon, J., \& Hicks, B. (2020). Characterising the Digital Twin: A systematic literature review. CIRP JMST, 29:36-52.

[9] Kalpic, B., \& Bernus, P. (2006). Business Process Modelling Through the Knowledge Management Perspective. Journal of Knowledge Management., 10(3):40-56.

[10] Lee, J., Bagheri, B., \& Kao, H.-A. (2015). A Cyber-Physical Systems architecture for Industry 4.0-based manufacturing systems. Manufacturing Letters, 3, 18-23.

[11] Lim, K. Y. H., Zheng, P., \& Chen, C.-H. (2020). A state-of-the-art survey of Digital Twin: techniques, engineering product lifecycle 
management and business innovation perspectives. J. Intell. Manuf., 31(6):1313-1337.

[12] Liu, Z, Meyendorf, N., Mrad, N. (2018). The role of data fusion in predictive maintenance using digital twin. Proc. AIP 1949, 020023, doi.org/10.1063/1.5031520

[13] Lu, Y., Morris, K., \& Frechette, S. (2016). Current Standards Landscape for Smart Manufacturing Systems. NISTIR 8107. Gaithersburg, MD, NIST.

[14] Mathis, C. (2017). Data Lakes. Datenbank-Spektrum, 17(3): 289293.

[15] Mitchell, W. (2010). Agile Sense-Making in the Battlespace. The Int. C2 Journal, 4(1):1-33.

[16] Negri, E., Fumagalli, L., \& Macchi, M. (2017). A review of the roles of Digital Twin in CPS-based production systems. Procedia Manufacturing, 11939 - 948.

[17] Niklasson L. et al. (2008). Extending the scope of situation analysis. In Proc. $11^{\text {th }}$ Int. Conf. on Information Fusion, pp1-8. Cologne, Germany

[18] Noran, O., Romero, D., \& Zdravkovic, M. (2014). The Sensing Enterprise: Towards the Next Generation Dynamic Virtual Organisations. IFIP Advances in Information and Communication Technology, 434:209-216

[19] Oks, S. J., Jalowski, M., Fritzsche, A., Möslein, K. M. (2019). Cyber-physical modeling and simulation: a reference architecture for designing demonstrators for industrial cyber-physical systems. Procedia CIRP, 84: 257-264.

[20] Pask, G. (1975). Conversation, Cognition and Learning. Elsevier, Amstaredam.

[21] Park, K.T., Son, Y.H., and Noh, S.D. (2020) The architectural framework of a cyber physical logistics system for digital-twinbased supply chain control. I. J. Prod. Res, 1:22 doi 10.1080/00207543.2020.1788738 (online July 2020)

[22] Selkowitz, A., Lakhmani, S., Chen, J. Y. C., Boyce, M. (2015). The Effects of Agent Transparency on Human Interaction with an Autonomous Robotic Agent. Proc the Factors and Ergonomics Society Annual Meeting, 59(1):806-810

[23] Van Brussel, H., Bongaerts, L., Wyns, J., Valckenaers, P., Van Ginderachter, T. (1999). A conceptual framework for holonic manufacturing: Identification of manufacturing holons. Journal of Manufacturing Systems, 18(1):35-52.

[24] van der Aalst, W., ter Hofstede, A. Weske, A.M.(Eds) (2003). Proc. Int. Conf. Business Process Management, Springer, Heidelberg.

\section{APPENDIX : EXPALANTION OF ISO15704/GERAM CONCEPTS USED IN THIS PAPER}

ISO15704/GERAM [6] is the result of over 25 years of effort by the IFIP-IFAC Task Force on Architectures for Enterprise Integration (in the period of 1990-2002) and subsequent work (during 1999-2019) by the International Standards Organisation (ISO) Working Group WG1 (Modelling and Architecture) under ISO/TC 184/SC5 (Interoperability, integration, and architectures for enterprise systems and automation applications).

The goal of developing GERAM was to systematise enterprise integration knowledge (as needed for example to create and to transform various enterprises, originally in the manufacturing domain, but with the finding that the result is universally applicable to a great variety of socio-technical systems of systems) [1]. Some essential concepts are discussed below:

Enterprise Entity: any entity that performs some useful system function or functions by providing a service or producing some goods. Example enterprise entities include a company, a project, a programme, a government, or government organisation, a product, a business unit, a manufacturing company, but also so-called virtual entities, such as a network of enterprises, or virtual organisations that only exist by way of a temporary or longer lasting alliance between other enterprise entities. Note that 'being a product' is relative, for example for an engineering company a factory may be its product.

An enterprise entity may have constituents (like subsystems in systems engineering), or it may exist as a system of systems ( $\mathrm{SoS}$ ) (when the contributing systems are not part of the SoS, they only provide services to the SoS).

Life cycle: this is a somewhat surprising concept of GERAM, because it is defined as a set of activity types, corresponding to the level of abstraction these activity types use to model the enterprise entity.

These activity types are also called life cycle 'phases'. A subdivision used in the definition of GERAM is identification, concept, requirements definition, preliminary design, detailed design, implementation / build, operation and decommissioning.

Care must be taken not to assume any temporal ordering whatsoever between these phases. After all these are only activity types and not activity instances, therefore information flow potentially exist in any direction among them. Time and the ordering of activity instances are described in the life history concept (below).

Life history: consists of life cycle activity instances. Any instance is an event, that is placed on the timeline, with a time to start and a time to finish. Typical sequences of events may be grouped into life history stages (which is identical to the concept of 'life cycle stage' used in Systems Engineering [5]), and stages may start and finish with a milestone. The life history of an entity at any one moment in time consists of one past history and (normally) several potential future histories.

Once the differentiation between life cycle and life history in this manner is understood, it dispels misunderstandings in communication among practitioners.

For example, the list of life cycle activity types (the 'phases') can be used to identify the competencies necessary to model or to design the entity, and in general to identify the responsibility of one entity in contributing to the life of another.

Also, transformation or development methodologies simply become patterns in the life history of an entity,. While the methodology may be based on a top-down waterfall progression, a bottom-up synthesis, an agile iteration, or a hybrid combination of these, it still remains true that competencies must be present to preform all (relevant) life cycle activity types. It is only a question when and how many times the instances of these types must be performed. This brings the discussion to the concept of life cycle relationships.

Life cycle relationship: an entity ' $A$ ' may contribute to the life cycle of another entity ' $\mathrm{B}$ ' by performing (or supporting) one or more life cycle activities, as illustrated in Fig. 3., including the fact the ' $A$ ' and ' $B$ ' may be the same entity. This type of life cycle relationship is called generative, because ' $\mathrm{A}$ ' contributes to the creation or change of entity ' $\mathrm{B}$ '.

As opposed to this, an operational life cycle relationship represents the fact that ' $A$ ' (as it operates) may support the operation of ' $\mathrm{B}$ '. 
Both of these relationships can be represented by arrows, where each arrow must start in the operation of entity, because after all an entity can only perform its services (fulfill its mission) as it operates. Long chains of such relationships may exist, as illustrated in Fig. 4.

Using these concepts a complex life history of any entity may be represented, such as the entity (e.g., a factory) may be operating, while at the same time a sequence of events plans and performs a longer duration factory refurbishment. Still another shorter sequence of events may be performing minor detailed design improvements, including decommissioning some equipment and (re)building (commissioning, configuring and deploying/ releasing into operation) some part.

Static and Dynamic Architecture: Part of the entity of interest must be pre-built and configured, thus implementing the entity's 'static architecture', with the aim of maintaining that structure over a longer period of time. As opposed to this part of the entity's structure must be adaptively (re)configured (to maintain the dynamic architecture), and this can be done using the capabilities that underly the "static architecture.

Modelling Framework vs Description Framework: GERAM has a modelling framework that may be imagined as a library of life cycle activity types and their outcomes in terms of models that represent the enterprise entity in question (see Fig. 2).

The shelves of this 'library' are labelled by the level (or extent) of abstraction on which the models occupying that shelf represent the entity. For example the Concept level holds all models that may be (or have been) produced to represent relevant facts about the entity, such as mission, vision, strategy, plans, policies and principles.

The scope of the models is subdivided in multiple orthogonal ways: e.g. the scope extends to i) everything automated vs everything implemented by humans; ii). models of the entity's mission fulfilment vs the models of the management and control of that entity; and iii) modelling aspects (function, information, resource and organisation), which are high level subdivisions, such that for example the 'function' aspect covers a large number of possible models.

Which one of these models is actually produced depends on the intent of the project architecting the entity and on associated stakeholder concerns (that can be answered through modelling, such as functional decomposition, activity models, behavioural models, simulation models, etc).

Note also, that models of increasing level of detail and decreasing level of abstraction would populate the concept, requirements, preliminary design and detailed design modelling levels.

Typically there can be many kinds of models, because the language in which the model is expressed would be selected to address some stakeholder concerns (which can be different from entity to entity).

In order to curb the complexity of models, it is useful to create more than one model, each representing some aspect of the entity, so that the collection of models on this level of abstraction is able to be used to address all relevant concerns that correspond to the stakeholder perspective.

While this arrangement is in line with a Model-based Systems Engineering (MBSE) or Enterprise-engineering philosophy, it is also true that stakeholders are often not familiar with the types of models that business analysts and enterprise architects use. Therefore the facts that can be projected as views of the underlying models may be presented using a 'user-friendly' formalism and be packaged as architecture descriptions, arranged in an architecture description framework.

In practice, given a domain of interest, several domainspecific architecture description frameworks have been developed (DoDAF, MODAF, UAF, ...), which capture typical stakeholder perspectives and aspects. These can be used in conjunction with the GERAM concepts and the organisation of its modelling framework, with the descriptions being views of one or more models. The discussion of these architecture description frameworks is beyond the scope of the present writing.

Note, though that typical description frameworks limit themselves to the extent of abstraction up to the preliminary design level, while GERAM's modelling framework covers all life cycle activity types up to and including operation (which is significant because models in a digital twin 'live there'), as well as decommissioning.

Note also, that the combination of the concepts of modelling framework and life history broadens the scope of architecture-related discussions, because every model on every level has its own temporal dimension (which is missing in current architecture description frameworks). 